\title{
Dynamical Behavior of a Nonautonomous Stochastic Modified Bazykin Model
}

\author{
Zhangzhi Wei $\mathbb{D}^{1,2}$ Zheng $W{ }^{1,}{ }^{1}$ and Lianglong Wang $\mathbb{D}^{1}$ \\ ${ }^{1}$ School of Mathematics and Statistics, Suzhou University, Suzhou 234000, China \\ ${ }^{2}$ School of Mathematical Sciences, Anhui University, Hefei 230601, China \\ Correspondence should be addressed to Lianglong Wang; wangll@ahu.edu.cn
}

Received 17 October 2019; Accepted 3 December 2019; Published 21 December 2019

Academic Editor: Juan L. G. Guirao

Copyright (C) 2019 Zhangzhi Wei et al. This is an open access article distributed under the Creative Commons Attribution License, which permits unrestricted use, distribution, and reproduction in any medium, provided the original work is properly cited.

In this article, a nonautonomous stochastic modified Bazykin model is introduced. The positive solution is proved to be unique and global for any initial data via stochastic comparison theorem and Itô formula. Stochastic ultimate boundedness and stochastic permanence are also considered. Finally, some simulations are performed to verify the validity of results.

\section{Introduction}

The study on population systems is one of the hot topic in applied mathematics and ecology. Mathematical modelling and qualitative analysis have received much concern, such as the famous Lotka-Volterra model [1]. For more than a decade, predator-prey models with ration-dependent response function have been discussed extensively [2-4]. Today, most predator-prey models assume that the functional response function is a function of prey population density [5-7].

Considering the classical assumptions is not always appropriate which assume that predators encounter prey at random, and the functional response only depends on prey abundance. In 1998, Alexeev and Bazykin first proposed the following Bazykin's model [2].

$$
\begin{aligned}
& \dot{N}(t)=N(t)\left(a-b N(t)-\frac{c P(t)}{1+A N(t)}\right), \\
& \dot{P}(t)=P(t)\left(-g-h P(t) \frac{f N(t)}{1+A N(t)}\right),
\end{aligned}
$$

where $N(t)$ and $P(t)$ denote the number (or density) of prey species and predator species. The parameters $a, b, c, g, f$, and $m$ are greater than zero with their usual ecological meanings (for details refer [2]). Many research studies about system (1) have been received, more about its generalization [8-11].

Taking the ratio-dependent of predator into account (more predators are good for hunting in serious situations), M. Haque remodeled a modified Bazykin model according to system (1)

$$
\begin{aligned}
& \dot{N}(t)=N(t)\left(a-b N(t)-\frac{c P(t)}{P(t)+A N(t)}\right), \\
& \dot{P}(t)=P(t)\left(-g-h P(t) \frac{f N(t)}{P(t)+A N(t)}\right),
\end{aligned}
$$

in which the analytical behavior of the system near origin is observed [1].

In the real environment, there is the interference of random factors everywhere, such as emergencies, weather changes, and disease spread, and then the actual observed data have some deviation from the results of the deterministic model. Therefore, the influence of the real random factors on the populations should be considered, and the stochastic model can describe the real situation better [12-16].

Considering that the parameters $a$ and $g$ are affected by white noise, the rates $a$ and $g$ can been replaced by $a \longrightarrow a+\sigma_{1} \dot{w}_{1}(t)$, and $g \longrightarrow g+\sigma_{2} \dot{w}_{2}(t)$, respectively, where $w_{1}(t)$ and $w_{2}(t)$ are Wiener process (or Brownian motion), and they are independent. $\sigma_{1}$ and $\sigma_{2}$ indicate the 
intensities of white noise. The following autonomous stochastic model was built by Lv et al. [13]:

$$
\begin{aligned}
& \dot{N}(t)=N(t)\left(a-b N(t)-\frac{c P(t)}{P(t)+A N(t)}\right)+\sigma_{1} N(t) \mathrm{d} w_{1}(t), \\
& \dot{P}(t)=P(t)\left(-g-h P(t) \frac{f N(t)}{P(t)+A N(t)}\right)-\sigma_{2} P(t) \mathrm{d} w_{2}(t),
\end{aligned}
$$

and has been investigated, and the system (3) is persistent in mean or die out under some conditions [13].

In this paper, we will consider the nonautonomous stochastic modified Bazykin model

$$
\begin{aligned}
\dot{N}(t)= & N(t)\left(a(t)-b(t) N(t)-\frac{c(t) P(t)}{P(t)+A(t) N(t)}\right) \\
& +\sigma_{1}(t) N(t) \mathrm{d} w_{1}(t), \\
\dot{P}(t)= & P(t)\left(-g(t)-h(t) P(t) \frac{f(t) N(t)}{P(t)+A(t) N(t)}\right) \\
& -\sigma_{2}(t) P(t) \mathrm{d} w_{2}(t),
\end{aligned}
$$

where $a(t), b(t), c(t), g(t), h(t), f(t), A(t)$, and $\sigma_{1}(t), \sigma_{2}(t)$ are assumed to be continuous and bounded.

It is important for population systems to keep permanence, which means that the species will not extinct in the future. Some definitions and conditions which guarantee that system (4) is stochastic permanent and boundedness are given as follows.

Without specification, $\left(N_{0}, P_{0}\right) \in R_{+}^{2}$ is an initial data by default in the full text, and $Y(t)=(N(t), P(t))$ is the solution of system (4) with an initial $\left(N_{0}, P_{0}\right)$.

Definition 1 (see [15]). Assume any $0<\epsilon<1$, then $M>0$ and $\eta>0$, when following is hold:

$$
\begin{gathered}
\liminf _{t \longrightarrow+\infty} \mathbb{P}\{|Y(t)| \leq M\} \geq 1-\epsilon, \\
\liminf _{t \longrightarrow+\infty} \mathbb{P}\{|Y(t)| \geq \eta\} \geq 1-\epsilon,
\end{gathered}
$$

so solution $Y(t)=(N(t), P(t))$ for system (4) is called stochastically permanent.

Definition 2 (see [15]). Assume any $0<\epsilon<1$, then $\eta>0$, when following is hold

$$
\underset{t \longrightarrow+\infty}{\lim \sup } \mathbb{P}\{|Y(t)|>\eta\}<\epsilon,
$$

so solution $Y(t)=(N(t), P(t))$ for system (4) is called stochastically ultimately bounded.

In Section 2, main results about the existence and uniqueness of global positive solution and the properties of asymptotic bounded were introduced and then shows us that the solution of system (4) is stochastically ultimate bounded and stochastically permanent. In Section 3, we briefly give conclusions and remark. In Section 4, numerical simulations are carried out to illustrate our results. In the end, some lemmas and proofs of main results are given.

\section{Main Results}

\subsection{Existence and Globality}

Theorem 1. Let $\left(N_{0}, P_{0}\right) \in R_{+}^{2}$ be initial value, then the positive solution $Y(t)=(N(t), P(t))$ to system (4) exists globally a.s.

Corollary 1. The solution $Y(t)=(N(t), P(t))$ of system (4) must stay in $R_{+}^{2}$ a.s., and that

$$
\begin{aligned}
& N_{1}(t) \leq N(t) \leq N_{2}(t), \\
& P_{1}(t) \leq P(t) \leq P_{2}(t), \quad \text { a.s., }
\end{aligned}
$$

where upper-lower solutions are defined later in (20)-(22) and (51).

2.2. Dynamical Behavior of System (4). In the following, we denote

$$
\begin{aligned}
\varphi^{u} & =\max _{t \in[0,+\infty)} \varphi(t), \\
\varphi_{l} & =\min _{t \in[0,+\infty)} \varphi(t), \\
q(t) & =a(t)-c(t)-\sigma_{1}^{2}(t), \\
\lambda(t) & =g(t)-\frac{f(t)}{A(t)}+\frac{1}{2} \sigma_{2}^{2}(t), \\
\nu(t) & =a(t)-c(t)-\frac{\sigma_{1}^{2}(t)}{2}, \\
\rho(t) & =a(t)-c(t)-\frac{3}{2} \sigma_{1}^{2}(t), \\
\sigma(t) & =\frac{f(t)}{A(t)}-g(t)-\frac{3}{2} \sigma_{2}^{2}(t) .
\end{aligned}
$$

The functions above are bounded and continuous on $[0,+\infty)$

Theorem 2. If $b_{l}>0$ and $g_{l}>0$, the solution $(N(t), P(t))$ to system (4) satisfies

$$
\limsup _{t \longrightarrow+\infty} \mathbb{E}[N(t)] \leq \frac{a^{u}}{b_{l}},
$$

$$
\underset{t \longrightarrow+\infty}{\limsup } \mathbb{E}\left[N(t)+\frac{c_{l}}{f^{u}} P(t)\right] \leq \frac{\left(a^{u}+g^{u}\right)^{2}}{4 b_{l} g_{l}} .
$$

Furthermore,

$$
\limsup _{t \longrightarrow+\infty} \mathbb{E}[P(t)] \leq \frac{f^{u}\left(a^{u}+g^{u}\right)^{2}}{4 b_{l} c_{l} g_{l}},
$$




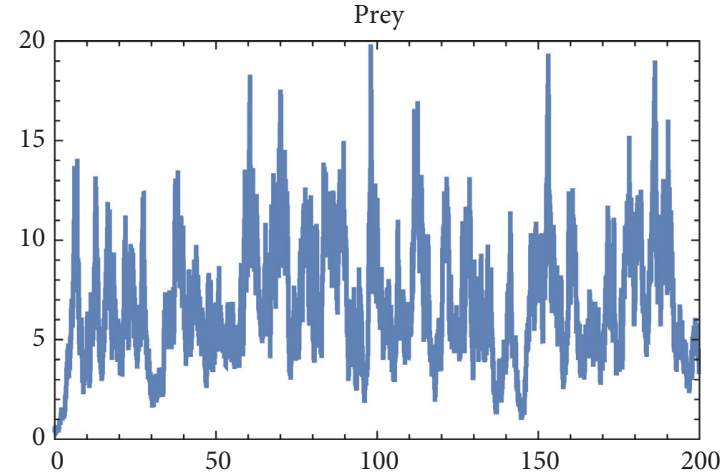

(a)

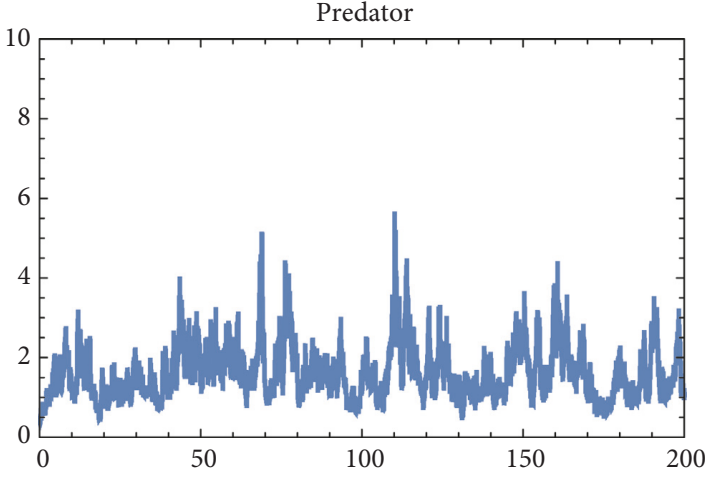

(b)

FIGURE 1: Solution is stochastically permanent if $b_{l}>0, c_{l}>0, g_{l}>0, r_{l}>0, \rho_{l}>0$, and $\sigma_{l}>0$.

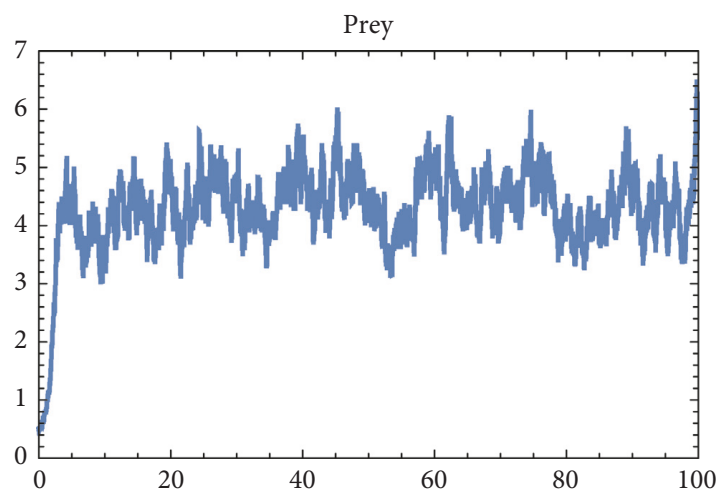

(a)

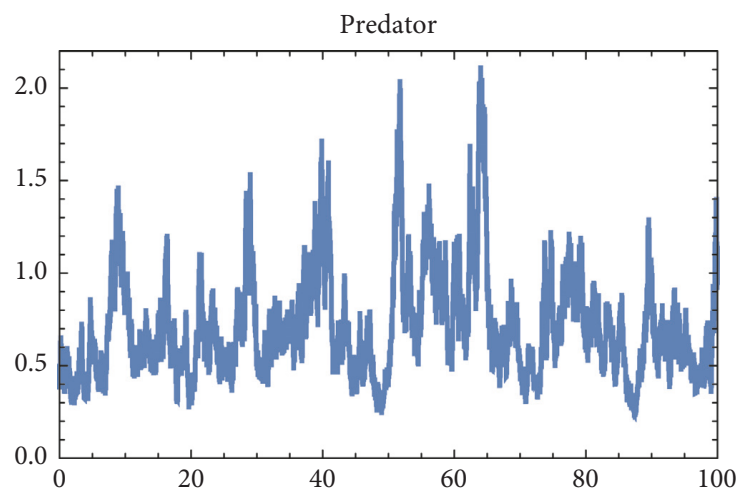

(b)

FIGURE 2: Solution is stochastically permanent if $b_{l}>0, c_{l}>0, g_{l}>0$, and $r_{l}>0$, but $\rho_{l}<0$ and $\sigma_{l}<0$.

when $c_{l}>0$.

Theorem 3. The solutions $(N(t), P(t))$ of system (4) are stochastically ultimately bounded when $b_{l}>0, c_{l}>0$, and $g_{l}>0$.

Theorem 4. The solutions $(N(t), P(t))$ of system (4) are stochastically permanent, if $b_{l}>0, c_{l}>0, g_{l}>0$, and $q_{l}>0$.

Theorem 5. The solutions $(N(t), P(t))$ of system (4) are stochastically permanent, if $b_{l}>0, c_{l}>0, g_{l}>0, \rho_{l}>0$, and $\sigma_{l}>0$.

\section{Conclusion}

Theorem 5 shows that predator species is permanent a.s., if $b_{l}>0, c_{l}>0, g_{l}>0, \rho_{l}>0$, and $\sigma_{l}>0$. It is clear that prey species is also permanent a.s., i.e., predator species and prey species are both stochastically permanent. Furthermore, under assumption of Theorem 5, system (4) is persistent according to Theorem 7 in [13]. By our Theorem 5, we can conclude that prey species and predator species in equation (4) are both stochastically permanent. Our work is based on the result of $\mathrm{Lv}$ et al. [13] and $\mathrm{Wu}$ et al. [15]. At the same time, we have achieved some results about the stochastic model with Markovian switching and Lévy jumps [17, 18].

\section{Simulations}

In this part, some numerical experiments are given to show that our main results as true. In Figure 1, we choose

$$
\begin{aligned}
a & =0.8+0.1 \sin t, \\
b & =0.1+0.05 \cos t \\
c & =0.2+\sin t \\
A & =0.9+0.1 \sin t \\
g & =0.3+0.01 \sin t \\
h & =0.1 \\
f & =0.9+0.1 \cos t \\
\sigma_{1} & =0.49+0.01 \sin t \\
\sigma_{2} & =0.49+0.1 \cos t
\end{aligned}
$$

We notice that $b_{l}>0, c_{l}>0, g_{l}>0, r_{l}>0$, $\rho_{l}>0$, and $\sigma_{l}>0$, and then the solution of system (4) will be stochastically permanent by virtue of Theorem 5 .

In Figure 2, we choose 


$$
\begin{aligned}
a & =0.8+0.1 \sin t, \\
b & =0.1+0.05 \cos t, \\
c & =0.2+\sin t, \\
A & =0.9+0.1 \sin t, \\
g & =0.3+0.01 \sin t, \\
h & =0.1, \\
f & =0.9+0.1 \cos t, \\
\sigma_{1} & =0.59+0.01 \sin t, \\
\sigma_{2} & =0.59+0.1 \cos t,
\end{aligned}
$$

where $r_{l}>0, \rho_{l}<0$, and $\sigma_{l}<0$, and $b_{l}>0, c_{l}>0, g_{l}>0$, and $r_{l}>0$, but $\rho_{l}<0$ and $\sigma_{l}<0$. According to Theorem 4, we know that the solution of system (4) is also stochastically permanent.

\section{Proofs of Main Results}

Lemma 1 (see [16]). Let $N_{2}(t)$ be a solution of equation (21). If $b_{l}>0$, then

$$
\limsup _{t \longrightarrow+\infty} \mathbb{E}\left[N_{2}(t)\right] \leq \frac{a^{u}}{b_{l}}
$$

Lemma 2 (see [16])

$$
\mathbb{E}\left[\exp \left\{\int_{t_{0}}^{t} \sigma_{1}(s) \mathrm{d} w(s)\right\}\right]=\exp \left\{\frac{1}{2} \int_{t_{0}}^{t} \sigma_{1}^{2}(s) \mathrm{d} s\right\}, \quad 0 \leq t_{0} \leq t .
$$

Lemma 3. If $q_{l}>0$, then

$$
\underset{t \rightarrow+\infty}{\limsup } \mathbb{E}\left[\frac{1}{N(t)}\right] \leq \frac{b^{u}}{q_{l}}
$$

Lemma 4. If $b_{l}>0$ and $q_{l}>0$, for every $\epsilon>0$, there exists $M=>0, \eta>0$; then,

$$
\begin{gathered}
\liminf _{t \longrightarrow+\infty} \mathbb{P}\{N(t) \leq M\} \geq 1-\epsilon, \\
\liminf _{t \longrightarrow+\infty} \mathbb{P}\{N(t) \geq \eta\} \geq 1-\epsilon .
\end{gathered}
$$

Lemma 5. If $\rho_{l}>0$ and $\sigma_{l}>0$, then

$$
\underset{t \longrightarrow+\infty}{\limsup } \mathbb{E}\left[\frac{1}{P(t)}\right] \leq P(0)^{-1}+\sqrt{f^{u} m^{u}\left[2 N(0)^{-1}+2\left(b^{u}\right)^{2} \rho_{l}^{-2}\right]} \text {. }
$$

Proof of Theorem 1 and Corollary 1. The proof process is a standard operation and is omitted here (see $[12,13,15])$.

The following system is obtained by comparison

$$
\begin{aligned}
\mathrm{d}_{1}(t)= & N_{1}(t)\left[a(t)-c(t)-b(t) N_{1}(t)\right] \mathrm{d} t \\
& +\sigma_{1}(t) N_{1}(t) \mathrm{d} w_{1}(t) \\
N_{1}(0)= & N(0) \\
\mathrm{d} N_{2}(t)= & N_{2}(t)\left[a(t)-b(t) N_{2}\right] \mathrm{d} t+\sigma_{1}(t) N_{2}(t) \mathrm{d} w_{1}(t), \\
N_{2}(0)= & N(0), \\
\mathrm{d} P_{1}(t)= & P_{1} t\left(\left(\frac{f(t)}{A(t)}-g(t)\right)-P_{1}(t)(h(t)\right. \\
& \left.\left.+\frac{f(t)}{A^{2}(t) P_{1}(t)}\right)\right) \mathrm{d} t-\sigma_{2}(t) P_{1}(t) \mathrm{d} w_{2}(t), \\
P_{1}(0)= & P(0),
\end{aligned}
$$$$
\begin{aligned}
\mathrm{d} P_{2}(t)= & P_{2}(t)\left(-h(t) P_{2}(t)+\left(\frac{f(t)}{A(t)}-g(t)\right)\right) \mathrm{d} t \\
& -\sigma_{2}(t) P_{2}(t) \mathrm{d} w_{2}(t), P_{2}(0)=P(0)
\end{aligned}
$$

They have solutions as follows:

$$
\begin{aligned}
& N_{1}(t)=\frac{\exp \left\{\int_{0}^{t}\left[a(s)-c(s)-\left(\sigma_{1}^{2}(s) / 2\right)\right] \mathrm{d} s+\int_{0}^{t} \sigma_{1}(s) \mathrm{d} w_{1}(s)\right\}}{N(0)^{-1}+\int_{0}^{t} b(s) \exp \left\{\int_{0}^{s}\left[a(\tau)-c(\tau)-\left(\sigma_{1}^{2}(\tau) / 2\right)\right] \mathrm{d} \tau+\int_{0}^{s} \sigma_{1}(\tau) \mathrm{d} w_{1}(\tau)\right\} \mathrm{d} s}, \\
& N_{2}(t)=\frac{\exp \left\{\int_{0}^{t}\left[a(s)-\left(\sigma_{1}^{2}(s) / 2\right)\right] \mathrm{d} s+\int_{0}^{t} \sigma_{1}(s) \mathrm{d} w_{1}(s)\right\}}{N(0)^{-1}+\int_{0}^{t} b(s) \exp \left\{\int_{0}^{s}\left[a(\tau)-\left(\sigma_{1}^{2}(\tau) / 2\right)\right] \mathrm{d} \tau+\int_{0}^{s} \sigma_{1}(\tau) \mathrm{d} w_{1}(\tau)\right\} \mathrm{d} s}, \\
& P_{1}(t)=\frac{\exp \left\{\int_{0}^{t}\left[(f(s) / A(s))-g(s)-\left(\sigma_{2}^{2}(s) / 2\right)\right] \mathrm{d} s-\int_{0}^{t} \sigma_{2}(s) \mathrm{d} w_{2}(s)\right\}}{P(0)^{-1}+\int_{0}^{t} h(s) \exp \left\{\int_{0}^{s}\left[(f(r) / A(r))-g(r)-\left(\sigma_{2}^{2}(s) / 2\right)\right] \mathrm{d} r-\int_{0}^{s} \sigma_{2}(r) \mathrm{d} w_{2}(r)\right\} \mathrm{d} s},
\end{aligned}
$$


$P_{2}(t)=\frac{\exp \left\{\int_{0}^{t}\left[(f(s) / A(s))-g(s)-\left(\sigma_{2}^{2}(s) / 2\right)\right] \mathrm{d} s-\int_{0}^{t} \sigma_{2}(s) \mathrm{d} w_{2}(s)\right\}}{P(0)^{-1}+\int_{0}^{t}\left(h(s)+\left(f(s) / A^{2}(s) N_{1}(s)\right)\right) \exp \left\{\int_{0}^{s}\left[(f(r) / A(r))-g(r)-\left(\sigma_{2}^{2}(s) / 2\right)\right] \mathrm{d} r-\int_{0}^{s} \sigma_{2}(r) \mathrm{d} w_{2}(r)\right\} \mathrm{d} s}$.

Consequently

$$
\begin{gathered}
N_{1}(t) \leq N(t) \leq N_{2}(t), \quad \text { a.s. } \\
P_{1}(t) \leq P(t) \leq P_{2}(t), \quad \text { a.s. }
\end{gathered}
$$

Proof of Theorem 2. From $N(t) \leq N_{2}(t)(t)$ a.s., and Lemma 1 , one obtain that

$$
\limsup _{t \longrightarrow+\infty} \mathbb{E}[N(t)] \leq \frac{a^{u}}{b_{l}} .
$$

Let

$$
F(t)=N(t)+\frac{c_{l}}{f^{u}} P(t)
$$

The derivative of $F(t)$ along system (4) can be obtained as follows:

$$
\begin{aligned}
\mathrm{d} F(t)= & \left((a(t)+g(t)) N(t)-b(t) N^{2}(t)-g(t) F(t)\right) \mathrm{d} t \\
& +\left(\left(-c(t)+\frac{c_{l}}{f^{u}} f(t)\right) \frac{N(t) P(t)}{y(t)+A(t) N(t)}-h(t) \frac{c_{l}}{f^{u}} P^{2}(t)\right) \mathrm{d} t \\
& +\sigma_{1}(t) N(t) \mathrm{d} w_{1}(t)-\frac{c_{l}}{f^{u}} \sigma_{2}(t) P(t) \mathrm{d} w_{2}(t) .
\end{aligned}
$$

Through calculating the integral, we have

$$
\begin{aligned}
F(t)= & F(0)+\int_{0}^{t}\left\{[a(s)+g(s)] N(s)-b(s) N^{2}(s)\right. \\
& -g(s) F(s)-h(t) \frac{c_{l}}{f^{u}} P^{2}(t)+\left(-c(s)+\frac{c_{l}}{f^{u}} f(s)\right) \\
& \left.\cdot \frac{N(s) P(s)}{P(s)+A(s) N(s)}\right\} \mathrm{d} s+\int_{0}^{t} \sigma_{1}(s) N(s) \mathrm{d} w_{1}(s) \\
& -\int_{0}^{t} \frac{c_{l}}{f^{u}} \sigma_{2}(s) \mathrm{d} w_{2}(s) .
\end{aligned}
$$

Then, we calculate the expectation

$$
\begin{aligned}
\mathbb{E}[F(t)]= & F(0)+\mathbb{E} \int_{0}^{t}\left\{[a(s)+g(s)] x(s)-b(s) N^{2}(s)\right. \\
& -g(s) F(s)-h(t) \frac{c_{l}}{f^{u}} P^{2}(t)+\left(-c(s)+\frac{c_{l}}{f^{u}} f(s)\right) \\
& \left.\cdot \frac{N(s) P(s)}{P(s)+A(s) N(s)}\right\} \mathrm{d} s .
\end{aligned}
$$

The following formula is easy to be obtained:

$$
\begin{aligned}
\frac{\mathrm{d} \mathbb{E}[F(t)]}{\mathrm{d} t}= & {[a(t)+g(t)] \mathbb{E}[N(t)]-b(t) \mathbb{E}\left[N^{2}(t)\right]-g(t) \mathbb{E}[F(t)]-h(t) \frac{c_{l}}{f^{u}} \mathbb{E}\left[P^{2}(t)\right] } \\
& +\left[-c(t)+\frac{c_{l}}{f^{u}} f(t)\right] \mathbb{E}\left[\frac{N(t) P(t)}{P(t)+A(t) N(t)}\right] \\
\leq & {[a(t)+g(t)] \mathbb{E}[N(t)]-b(t) \mathbb{E}\left[N^{2}(t)\right]-g(t) \mathbb{E}[F(t)] } \\
\leq & \left(a^{u}+g^{u}\right) \mathbb{E}[N(t)]-b_{l}(\mathbb{E}[N(t)])^{2}-g_{l} \mathbb{E}[F(t)] .
\end{aligned}
$$

Note that inequality $K_{1} x-K_{2} x^{2} \leq K_{1}^{2} / 4 K_{2}$, and then

$$
\frac{\mathrm{d} \mathbb{E}[F(t)]}{\mathrm{d} t} \leq \frac{\left(a^{u}+g^{u}\right)^{2}}{4 b_{l}}-g_{l} \mathbb{E}[F(t)]
$$

Therefore, by basic theories of ordinary differential equation, we have

$$
0 \leq \limsup _{t \longrightarrow+\infty} \mathbb{E}[F(t)] \leq \frac{\left(a^{u}+g^{u}\right)^{2}}{4 b_{l} g_{l}} .
$$

According to the above equation, the following is obtained:

$$
\limsup _{t \longrightarrow+\infty} \mathbb{E}[P(t)] \leq-\mathbb{E}[N(t)]+\frac{f^{u}\left(a^{u}+g^{u}\right)^{2}}{4 b_{l} c_{l} g_{l}} \leq \frac{f^{u}\left(a^{u}+g^{u}\right)^{2}}{4 b_{l} c_{l} g_{l}} .
$$

Proof of Theorem 3. By Theorem 2,

$$
\begin{aligned}
& \limsup _{t \rightarrow+\infty} \mathbb{E}[N(t)] \leq \frac{a^{u}}{b_{l}}, \\
& \limsup _{t \longrightarrow+\infty} \mathbb{E}[P(t)] \leq \frac{f_{l}\left(a^{u}+g^{u}\right)^{2}}{4 b_{l} c_{l} g_{l}} .
\end{aligned}
$$


Now, for any $\epsilon>0$, let $H_{1}>\left(a^{u} / b_{l} \epsilon\right)$ and $H_{2}>\left(\left(a^{u}+g^{u}\right)^{2} f^{u} / 4 b_{l} c_{l} g_{l} \epsilon\right)$. Then, the following are obtained through the Chebyshev inequality

$$
\begin{gathered}
\mathbb{P}\left\{N(t)>H_{1}\right\} \leq \frac{\mathbb{E}[N(t)]}{H_{1}}<\epsilon, \\
\mathbb{P}\left\{P(t)>H_{2}\right\} \leq \frac{\mathbb{E}[P(t)]}{H_{2}}<\epsilon .
\end{gathered}
$$

Taking $H=2 \max \left\{H_{1}, H_{2}\right\}$, it yields

$$
\mathbb{P}\{|Y(t)|>H\} \leq \mathbb{P}\{N(t)+P(t)>H\} \leq \frac{\mathbb{E}[N(t)+P(t)]}{H}<\epsilon .
$$

Then,

$$
\limsup _{t \longrightarrow+\infty} \mathbb{P}\{|Y(t)|>H\}<\epsilon .
$$

Proof of Lemma 3. Combing (21) with Lemma 2, we have

$$
\begin{aligned}
E\left[\frac{1}{N_{1}(t)}\right]= & N(0)^{-1} \exp \left\{-\int_{0}^{t}\left[a(s)-c(s)-\frac{\sigma_{1}^{2}(s)}{2}\right] \mathrm{d} s\right\} \mathbb{E}\left[\exp \left\{-\int_{0}^{t} \sigma_{1}(s) \mathrm{d} w_{1}(s)\right\}\right] \\
& +\int_{0}^{t} b(s) \exp \left\{-\int_{s}^{t}\left[a(\tau)-c(\tau)-\frac{\sigma_{1}^{2}(\tau)}{2}\right] \mathrm{d} \tau\right\} \mathbb{E}\left[\exp \left\{-\int_{s}^{t} \sigma_{1}(\tau) \mathrm{d} w_{1}(\tau)\right\}\right] \mathrm{d} s \\
= & N(0)^{-1} \exp \left\{-\int_{0}^{t} q(s) \mathrm{d} s\right\}+\int_{0}^{t} b(s) \exp \left\{-\int_{s}^{t} q(\tau) \mathrm{d} \tau\right\} \mathrm{d} s \\
\leq & N(0)^{-1} e^{-q_{l} t}+b^{u} \int_{0}^{t} e^{-q_{l}(t-s)} \mathrm{d} s \leq N(0)^{-1} e^{-q_{l} t}+\frac{b^{u}}{q_{l}} .
\end{aligned}
$$

From Corollary 1, it has

$$
\mathbb{E}\left[\frac{1}{N(t)}\right] \leq \mathbb{E}\left[\frac{1}{N(t)}\right] \leq N(0)^{-1} e^{-q_{l} t}+\frac{b^{u}}{q_{l}} .
$$

Therefore, $\lim \sup _{t \rightarrow+\infty} \mathbb{E}[1 / N(t)] \leq\left(b^{u} / q_{l}\right)$.

Proof of Lemma 4. By Theorem 2, there exists $K>0$ s.t. $\mathbb{E}[N(t)] \leq K$. For each $\epsilon>0$, denote $H=K / \epsilon$; hence, we have

$$
\mathbb{P}\{N(t)>H\} \leq \frac{\mathbb{E}[N(t)]}{H} \leq \epsilon .
$$

Then,

$$
\mathbb{P}\{N(t) \leq H\} \geq 1-\epsilon .
$$

By Lemma 3, we have

$$
\limsup _{t \longrightarrow+\infty} \mathbb{E}\left[\frac{1}{N(t)}\right] \leq \frac{b^{u}}{q_{l}} .
$$

Therefore, for any $\epsilon>0$, let $\delta=\epsilon r_{l} / b^{u}$, we have

$$
\mathbb{P}\{N(t)<\delta\}=\mathbb{P}\left\{\frac{1}{N(t)}>\frac{1}{\delta}\right\} \leq \frac{\mathbb{E}[1 / N(t)]}{1 / \delta} \leq \delta \mathbb{E}[1 / N(t)],
$$

which yields

$$
\limsup _{t \rightarrow+\infty} \mathbb{P}[N(t)<\delta] \leq \delta \mathbb{E}\left[\frac{1}{N(t)}\right]=\frac{\delta b^{u}}{q_{l}}=\frac{\epsilon q_{l}}{b^{u}} \cdot \frac{b^{u}}{q_{l}}=\epsilon .
$$

This implies

$$
\liminf _{t \longrightarrow+\infty} \mathbb{P}[N(t) \geq \delta] \geq 1-\epsilon .
$$

Proof of Theorem 4. According to the arguments in Lemma 4 , we know that for any $\epsilon$, existing $\delta>0, M>0$ and the following is true:

$$
\liminf _{t \longrightarrow+\infty} \mathbb{P}\{N(t) \geq \delta\} \geq 1-\epsilon .
$$

Then, we get

$$
\liminf _{t \longrightarrow+\infty} \mathbb{P}\{|Y(t)| \geq \delta\} \geq \liminf _{t \longrightarrow+\infty} \mathbb{P}\{N(t) \geq \delta\} \geq 1-\epsilon .
$$

Similarly, we have

$$
\liminf _{t \longrightarrow+\infty} \mathbb{P}\{|Y(t)| \leq M\} \geq 1-\epsilon .
$$

From Definition 1, we know the solution of system (4) is stochastically permanent.

Proof of Lemma 5. By Corollary 1, it is easy to have 


$$
\begin{aligned}
& P_{1}(t)<P(t), \\
& P_{1}(t)=\frac{\exp \left\{\int_{0}^{t}\left[(f(s) / A(s))-g(s)-\left(\sigma_{2}^{2}(s) / 2\right)\right] \mathrm{d} s-\int_{0}^{t} \sigma_{2}(s) \mathrm{d} w_{2}(s)\right\}}{P(0)^{-1}+\int_{0}^{t}\left[h(s)+\left(f(s) / A^{2}(s) \phi(s)\right)\right] \exp \left\{\int_{0}^{s}\left[(f(r) / A(r))-g(r)-\left(\sigma_{2}^{2}(s) / 2\right)\right] \mathrm{d} r-\int_{0}^{s} \sigma_{2}(r) \mathrm{d} w_{2}(r)\right\} \mathrm{d} s} .
\end{aligned}
$$

So,

$$
\begin{aligned}
P_{1}^{-1}(t)= & P(0)^{-1} \exp \left\{\int_{0}^{t}\left[-\frac{f(s)}{A(s)}+g(s)+\left(\sigma_{2}^{2} \frac{(s)}{2}\right)\right] \mathrm{d} s+\int_{0}^{t} \sigma_{2}(s) \mathrm{d} w_{2}(s)\right\} \\
& +\int_{0}^{t}\left(h(s)+\frac{f(s)}{A^{2}(s) \phi(s)}\right) \exp \left\{\int_{s}^{t}\left[g(\tau)-\frac{f(\tau)}{A(\tau)}+\frac{1}{2} \sigma_{2}^{2}(\tau)\right] \mathrm{d} \tau+\int_{s}^{t} \sigma_{2}(\tau) \mathrm{d} w_{2}(\tau)\right\} \mathrm{d} s .
\end{aligned}
$$

By Lemma 2 and Hölder inequality, one has

$$
\begin{aligned}
\mathbb{E}\left[P_{1}^{-1}(t)\right] \leq & P(0)^{-1} \exp \left\{\int_{0}^{t}\left[g(s)-\frac{f(s)}{A(s)}+\sigma_{2}^{2}(s)\right] \mathrm{d} s\right\} \\
& +\int_{0}^{t}\left[h(s)+\frac{f(s)}{A^{2}(s) \phi(s)}\right] \exp \left\{\int_{s}^{t}[d(\tau)\right. \\
& \left.\left.-f(\tau)+\frac{3}{2} \sigma_{2}^{2}(\tau)\right] \mathrm{d} \tau\right\}\left\{\mathbb{E}\left[\phi^{-2}(s)\right]\right\}^{1 / 2} \mathrm{~d} s .
\end{aligned}
$$

Combiing inequality $(a+b)^{2} \leq 2\left(a^{2}+b^{2}\right)$ with (21), one can get

$$
\begin{aligned}
\mathbb{E}\left[N_{1}^{-2}(t)\right] \leq & 2 x_{0}^{-2} \mathbb{E}\left\{\exp \left\{-2 \int_{0}^{t} \nu(s) \mathrm{d} s-2 \int_{0}^{t} \sigma_{1}(s) \mathrm{d} w_{1}(s)\right\}\right\} \\
& +2 \mathbb{E}\left\{\int_{0}^{t} b(s) \exp \left\{-\int_{s}^{t} \nu(\tau) \mathrm{d} \tau-\int_{s}^{t} \sigma_{1}(\tau) \mathrm{d} w_{1}(\tau)\right\} \mathrm{d} s\right\}^{2} .
\end{aligned}
$$

Meanwhile,

$$
\begin{aligned}
& \mathbb{E}\left\{\int_{0}^{t} b(s) \exp \left\{-\int_{s}^{t} \nu(\tau) \mathrm{d} \tau-\int_{s}^{t} \sigma_{1}(\tau) \mathrm{d} w_{1}(\tau)\right\} \mathrm{d} s\right\}^{2} \\
& =\int_{0}^{t} \int_{0}^{t} b(s) b(u) \exp \left\{-\int_{s}^{t} \nu(\tau) \mathrm{d} \tau\right\} \exp \left\{-\int_{s}^{t} \nu(\tau) \mathrm{d} \tau\right\} \\
& \cdot \mathbb{E}\left[\exp \left\{-\int_{s}^{t} \sigma_{1}(\tau) \mathrm{d} w_{1}(\tau)\right\} \exp \left\{-\int_{u}^{t} \sigma_{1}(\tau) \mathrm{d} w_{1}(\tau)\right\}\right] \mathrm{d} u \mathrm{~d} s .
\end{aligned}
$$

Furthermore,

$$
\begin{aligned}
& \mathbb{E}\left[\exp \left\{-\int_{s}^{t} \sigma_{1}(\tau) \mathrm{d} w_{1}(\tau)\right\} \exp \left\{-\int_{u}^{t} \sigma_{1}(\tau) \mathrm{d} w_{1}(\tau)\right\}\right] \\
& \leq \exp \left\{\int_{s}^{t} \sigma_{1}^{2}(\tau) \mathrm{d} \tau\right\} \exp \left\{\int_{u}^{t} \sigma_{1}^{2}(\tau) \mathrm{d} \tau\right\} .
\end{aligned}
$$

Substituting (53) into (52), we have

$$
\begin{aligned}
& \mathbb{E}\left\{\int_{0}^{t} b(s) \exp \left\{-\int_{s}^{t} \nu(\tau) \mathrm{d} \tau-\int_{s}^{t} \sigma_{1}(\tau) \mathrm{d} w_{1}(\tau)\right\} \mathrm{d} s\right\}^{2} \\
& =\left\{\int_{0}^{t} b(s) \exp \left\{-\int_{s}^{t}\left[a(\tau)-\frac{c(\tau)}{m(\tau)}-\frac{3}{2} \sigma_{1}^{2}(\tau)\right] \mathrm{d} \tau\right\} \mathrm{d} s\right\}^{2} .
\end{aligned}
$$

Therefore, by (51) and (54), one can get

$$
\begin{aligned}
\mathbb{E}\left[N_{1}^{-2}(t)\right] & \leq \frac{2}{N(0)} \exp \left\{-2 \rho_{l} t\right\}+2\left(\frac{b^{u}}{\rho_{l}}\right)^{2} \\
& \leq \frac{2}{N(0)}+2\left(\frac{b^{u}}{\rho_{l}}\right)^{2} .
\end{aligned}
$$

Finally, we obtain the required assertion through substituting (55) into (51).

In summary, Theorem 5 has been obtained.

Remark 1. It is clear that stochastic differential equation which is stochastically permanent implies that its solutions are stochastically ultimately bounded. Lemma 4 shows that the prey species will be permanent if $b_{l}>0$ and $q_{l}>0$, or it will be extinct. Theorem 4 shows that if $b_{l}>0, c_{l}>0, g_{l}>0$ and $q_{l}>0$, system (4) is permanent in probability 1 , i.e., the number (or density) is bounded a.s.

\section{Data Availability}

The data used to support this study are provided in our paper.

\section{Conflicts of Interest}

The authors declare that they have no conflicts of interest.

\section{Acknowledgments}

This work was supported by the Natural Science Foundation of China (no. 11771001), Provincial Natural Science Research Project of Anhui Colleges (nos. KJ2019A0672 and 
KJ2019A0666), Program for Excellent Young Talents in University of Anhui Province (no. gxyq2017092), and Key Teaching Research Project of Suzhou University (no. szxy2018jyxm12).

\section{References}

[1] M. Haque, "Ratio-dependent predator-prey models of interacting populations," Bulletin of Mathematical Biology, vol. 71, no. 2, pp. 430-452, 2009.

[2] A. D. Bazykin, A. I. Khibnik, and B. Krauskopf, Nonlinear Dynamics of Interacting Populations, World Scientific, Singapore, 1998.

[3] Y. Kuang and E. Beretta, "Global qualitative analysis of a ratiodependent predator-prey system," Journal of Mathematical Biology, vol. 36, no. 4, pp. 389-406, 1998.

[4] H. R. Akcakaya, R. Arditi, and L. R. Ginzburg, "Ratio-dependent predation: an abstraction that works," Ecology, vol. 76, no. 3, pp. 995-1004, 1995.

[5] H.-B. Shi, S. Ruan, Y. Su, and J.-F. Zhang, "Spatiotemporal dynamics of a diffusive Leslie-Gower predator-prey model with ratio-dependent functional response," International Journal of Bifurcation and Chaos, vol. 25, no. 5, Article ID 1530014, 2015.

[6] J. Zhou, "Bifurcation analysis of a diffusive predator-prey model with ratio-dependent Holling type III functional response," Nonlinear Dynamics, vol. 81, no. 3, pp. 1535-1552, 2015.

[7] C. M. Heggerud and K. Lan, "Local stability analysis of ratiodependent predator-prey models with predator harvesting rates," Applied Mathematics and Computation, vol. 270, pp. 349-357, 2015.

[8] M. Sen, M. Banerjee, and A. Morozov, "Bifurcation analysis of a ratio-dependent prey-predator model with the Allee effect," Ecological Complexity, vol. 11, pp. 12-27, 2012.

[9] M. Banerjee and S. Abbas, "Existence and non-existence of spatial patterns in a ratio-dependent predator-prey model," Ecological Complexity, vol. 21, pp. 199-214, 2015.

[10] C. Çelik, "Stability and Hopf bifurcation in a delayed ratio dependent Holling-Tanner type model," Applied Mathematics and Computation, vol. 255, pp. 228-237, 2015.

[11] D. Xiao and L. S. Jennings, "Bifurcations of a ratio-dependent predator-prey system with constant rate harvesting," SIAM Journal on Applied Mathematics, vol. 65, no. 3, pp. 737-753, 2005.

[12] C. Ji, D. Jiang, and X. Li, "Qualitative analysis of a stochastic ratio-dependent predator-prey system," Journal of Computational and Applied Mathematics, vol. 235, no. 5, pp. 13261341, 2011.

[13] J. Lv, K. Wang, and D. Chen, "Analysis on a stochastic twospecies ratio-dependent predator-prey model," Methodology and Computing in Applied Probability, vol. 17, no. 2, pp. 403-418, 2015.

[14] X. Mao, Stochastic Differential Equations and Their Applications, Horwood Publishing, Chichester, UK, 2007.

[15] Z. Wu, H. Huang, and L. Wang, "Dynamical behavior of a stochastic ratio-dependent predator-prey system," Journal of Applied Mathematics, vol. 2012, Article ID 857134, 17 pages, 2012.

[16] D. Jiang and N. Shi, "A note on nonautonomous logistic equation with random perturbation," Journal of Mathematical Analysis and Applications, vol. 303, no. 1, pp. 164-172, 2005.

[17] Z. Wei, Z. Wu, L. Hu, and L. Wang, "Stochastic modified Bazykin predator-prey model with Markovian switching,"
International Journal of Nonlinear Sciences and Numerical Simulation, vol. 19, no. 6, pp. 573-581, 2018.

[18] Z. Wei, Z. Wu, L. Hu, and L. Wang, "Persistence and extinction of a stochastic modified Bazykin predator-prey system with Lévy jumps," Discrete Dynamics in Nature and Society, vol. 2018, Article ID 8479101, 7 pages, 2018. 

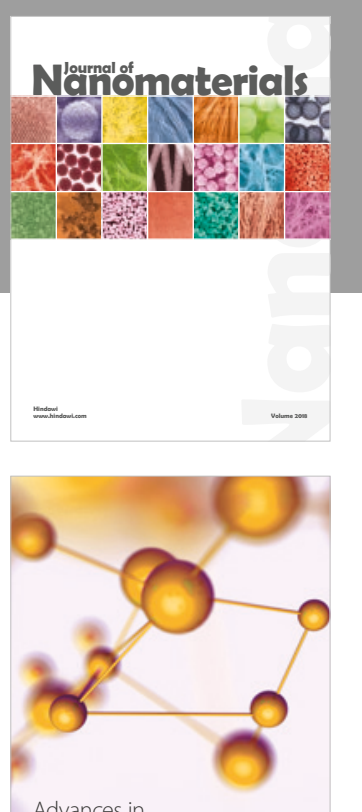

Physical Chemistry
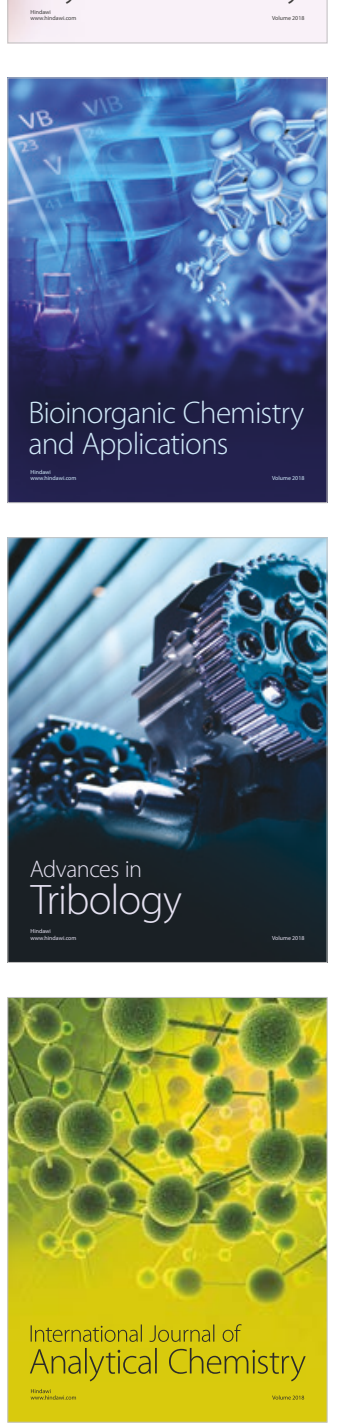

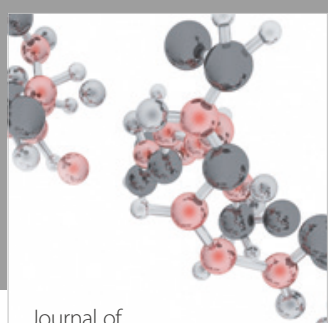

Analytical Methods

in Chemistry

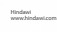

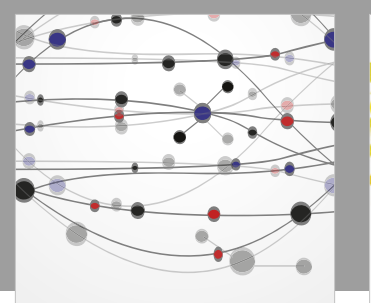

The Scientific World Journal

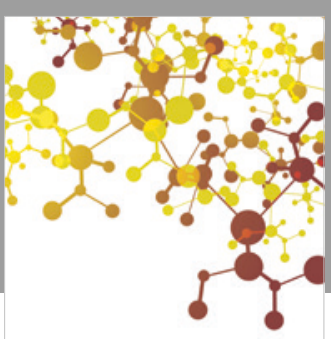

Journal of

Applied Chemistry
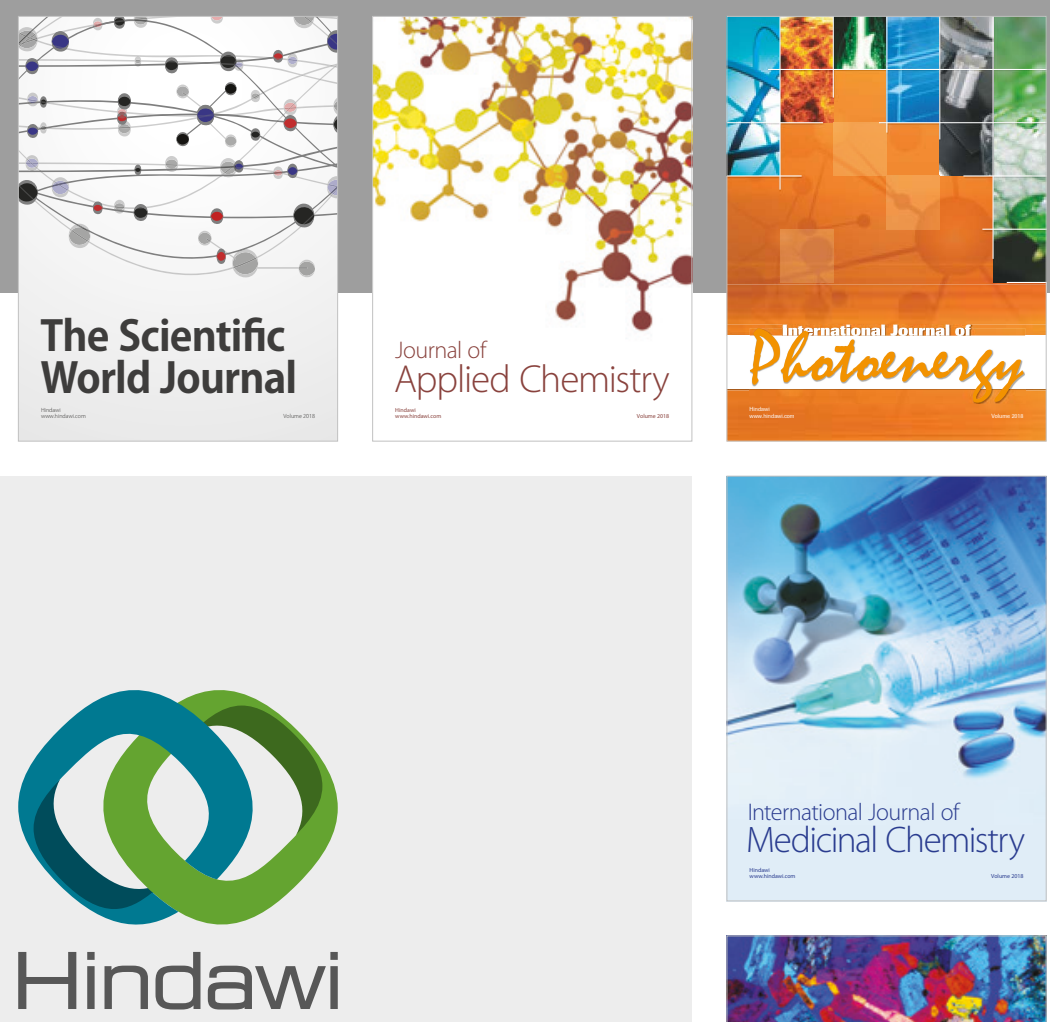

Submit your manuscripts at

www.hindawi.com
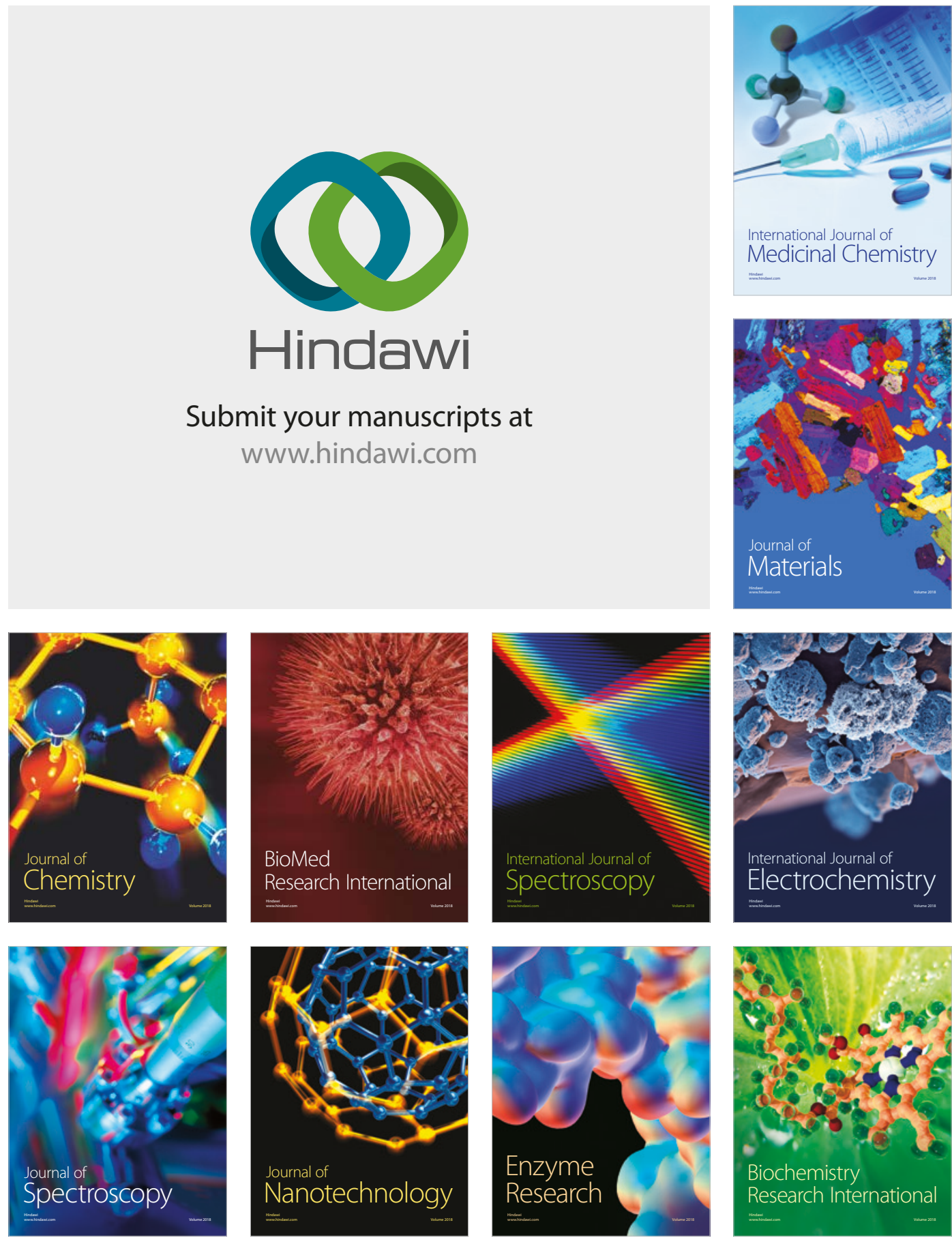
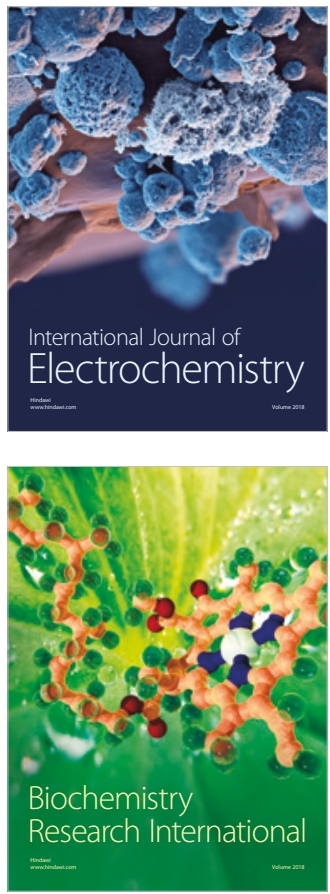